agreements with Karolinska Institutet with JA as principal investigator, mainly in the context of safety monitoring of biologics via the ARTIS national safety monitoring system

DOI: 10.1136/annrheumdis-2021-eular.2626

\begin{tabular}{|l|l}
\hline OP0115 & EFFICACY AND SAFETY OF ABBV-3373, A NOVEL \\
ANTI-TNF GLUCOCORTICOID RECEPTOR \\
MODULATOR ANTIBODY DRUG CONJUGATE, \\
IN PATIENTS WITH MODERATE TO SEVERE \\
RHEUMATOID ARTHRITIS DESPITE METHOTREXATE \\
THERAPY: A PHASE 2A PROOF OF CONCEPT STUDY
\end{tabular}

F. Buttgereit $^{1}$, J. Aelion ${ }^{2}$, B. Rojkovich ${ }^{3}$, A. Zubrzycka-Sienkiewicz ${ }^{4}$, T. Radstake ${ }^{5}$, S. Chen ${ }^{5}$, D. Arikan ${ }^{5}$, H. Kupper ${ }^{6}$, H. Amital ${ }^{7,8} .{ }^{1}$ Charité University Medicine, Department of Rheumatology and Clinical Immunology, Berlin, Germany; ${ }^{2}$ West Tennessee Research Institute, Jackson, United States of America; ${ }^{3}$ Buda Hospital of the Hospitaller Order of St. John of God, Rheumatology, Budapest, Hungary; ${ }^{4}$ Reumatika -Centrum Reumatologii, ARS Rheumatica sp.z.o.o, Warsaw, Poland; ${ }^{5}$ AbbVie, Immunology, North Chicago, United States of America; ${ }^{6}$ AbbVie Deutschland GmbH \& Co. KG, Immunology, Ludwigshafen, Germany; ${ }^{7}$ Sheba Medical Center, Department of Medicine ' $B$ ' and Zabludowicz Center for Autoimmune Diseases, Ramat Gan, Israel; ${ }^{8}$ Affiliated to Sackler Faculty of Medicine, Tel Aviv University, Tel Aviv, Israel

Background: ABBV-3373 is a novel antibody drug conjugate composed of adalimumab (ADA) linked to a proprietary and highly potent glucocorticoid receptor modulator (the anti-inflammatory payload) currently evaluated for the treatment of rheumatoid arthritis (RA).

Objectives: To assess the efficacy and safety of ABBV-3373 vs ADA in RA patients (pts).

Methods: This was a 24-week (wk) randomized, double-blind, double-dummy, active-controlled Phase 2a study of intravenously (IV)-administered ABBV-3373 $100 \mathrm{mg}$ (for 12 wks followed by placebo [PBO] for $12 \mathrm{wks}$ ) vs subcutaneous injections of ADA $80 \mathrm{mg}$ every other wk (for $24 \mathrm{wks}$ ) in pts on background methotrexate. The primary endpoint was the change from baseline (BL) in DAS28(CRP) at Wk 12. Pre-planned statistical methods incorporating pre-specified historical ADA data both alone (pre-specified success criterion, 2-sided $P \leq 0.1$ ) and supplemented with in-trial ADA data (pre-specified success criterion, probability $>95 \%$ ) were used to achieve adequate statistical power with a reduced trial size. Assay sensitivity was evaluated through construction of a synthetic PBO arm by propensity score matching, using individual pt-level PBO data from 3 recent sponsor-run trials of similar populations and trial settings. Secondary endpoints at Wk 12 included 1) mean change from BL in CDAI, SDAI, DAS28(ESR), HAQ-DI; 2) proportion of pts achieving DAS28(CRP) $\leq 3.2, \quad$ ACR20/50/70 responses, $\mathrm{HAQ}-\mathrm{DI} \leq-0.22$. Continuous and categorical efficacy variables were analyzed using mixed effect model repeated measurements and Cochran-Mantel-Haenszel test, respectively; non-responder imputation was applied to missing categorical data. Treatment-emergent adverse events were summarized through Wk 12

Results: A total of 48 pts were randomized and treated (ABBV-3373: 31; ADA: $17) ; 46$ pts $(96 \%)$ completed 12 wks of study treatment. BL demographics and disease characteristics were indicative of established RA and similar among the 2 treatment arms and the synthetic PBO arm. ABBV-3373 demonstrated significant improvement in mean DAS28(CRP) at Wk 12 vs the pre-specified historical ADA (-2.65 vs $-2.13 ; P=0.022)$ and numerically greater improvement vs the combined in-trial and historical ADA arm (-2.65 vs -2.29 ; probability $90 \%$; Figure). Comparable improvements in disease activity and targets were observed for ABBV-3373 and in-trial ADA. Assay sensitivity was supported by the fact that both ABBV-3373 and ADA arms were superior to synthetic PBO $(P<0.001)$. For secondary endpoints, greater efficacy was observed with ABBV-3373 vs historical

Table 1. Treatment Emergent Adverse Events up to Week 12

\begin{tabular}{lcc}
\hline Event, $\mathbf{n}(\%)$ & $\begin{array}{c}\text { ADA } \\
(\mathbf{N}=\mathbf{1 7})\end{array}$ & $\begin{array}{c}\text { ABBV-3373 } \\
\mathbf{( N = 3 1 )}\end{array}$ \\
\hline Adverse event (AE) & $12(70.6)$ & $11(35.5)$ \\
AE with reasonable possibility of being drug related & $3(17.6)$ & $2(6.5)$ \\
Severe AE & 0 & $1(3.2)$ \\
Serious AE & 0 & $4(12.9)^{\#}$ \\
AE leading to Discontinuation of Study Drug & $1(5.9)$ & $1(3.2)$ \\
Serious infections & 0 & $2(6.5)$ \\
Opportunistic infection excluding Tuberculosis & 0 & 0 \\
Allergic Reactions Including Hypersensitivity, & $2(11.8)^{\text {\& }}$ & $1(3.2)^{\wedge}$ \\
Angioedema, and Anaphylaxis & 0 & 0 \\
Systemic glucocorticoid events & 0 & 0 \\
All deaths & 0 & 0 \\
\$As assessed by investigator.\#Serious AEs: 1 non-cardiac chest pain, 1 pneumonia, 1 upper
\end{tabular}

${ }^{\$}$ As assessed by investigator. "Serious AEs: 1 non-cardiac chest pain, 1 pneumonia, 1 upp
respiratory tract disease and 1 anaphylactic shock. ${ }^{*} 1$ Type I hypersensitivity, 1 Pruritus 11 Anaphylactic shock
ADA; ABBV-3373 was predicted with $79-99 \%$ probability to be better than ADA based on the combined in-trial and historical ADA data. 2 serious infections were reported with $A B B V-3373$ (pneumonia, upper respiratory tract infection) and none with ADA through Wk 12 (Table). 1 event of anaphylactic shock reaction was reported with ABBV-3373. After increasing the duration of IV administration from $3 \mathrm{~min}$ to $15-30 \mathrm{~min}$, no similar events were observed.

Conclusion: These data demonstrate the clinical efficacy of ABBV-3373 and its potential to provide improved outcomes for RA pts compared to ADA. The safety profile of ABBV-3373 was generally similar to ADA.

Acknowledgements: AbbVie and the authors we thank the patients, trial sites, and investigators who participated in this clinical trial. AbbVie, Inc was the trial sponsor, contributed to trial design, data collection, analysis \& interpretation, and to writing, reviewing, and approval of final version. No honoraria or payments were made for authorship. The authors thank Yang Yang of AbbVie Inc for supporting the statistical analysis and data reporting. Medical writing support was provided by Ramona Vladea, PhD of AbbVie, Inc.

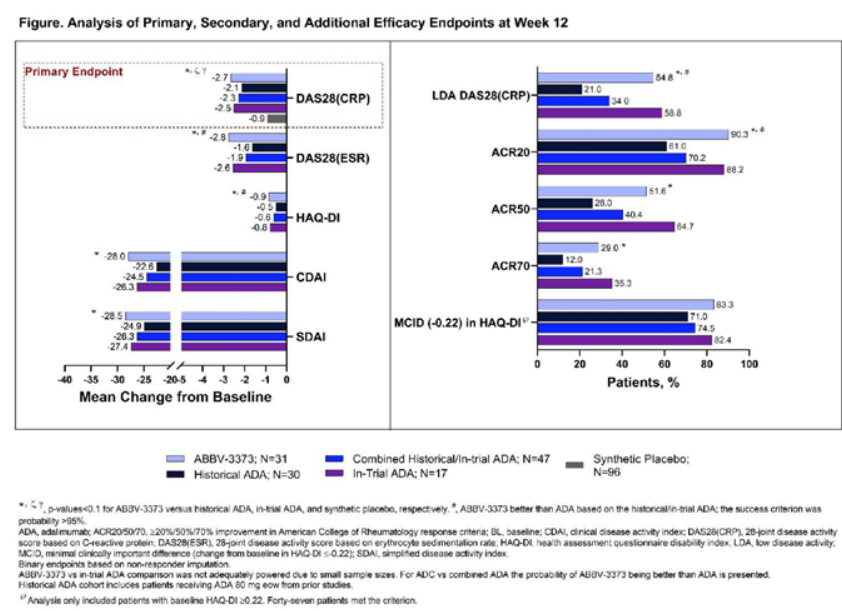

Disclosure of Interests: Frank Buttgereit Consultant of: AstraZeneca, AbbVie Grünenthal, Horizon Pharma, Pfizer, and Roche, Grant/research support from: AbbVie, Horizon Pharma, Pfizer, and Roche, Jacob Aelion Grant/research support from: AbbVie, Amgen, AstraZeneca, BMS, Celgene, Eli Lilly, Galapagos/ Gilead, Genentech, GlaxoSmithKline, Horizon, Janssen, Mallinckrodt, Nektar Nichi-Iko, Novartis, Pfizer, Regeneron, Roche, Sanofi-Aventis, Selecta, UCB Bernadette Rojkovich: None declared, Anna Zubrzycka-Sienkiewicz Consultan of: Astellas and Roche, Grant/research support from: AbbVie, Astellas, Galapagos NV, Gilead Sciences, Janssen, Lilly, Mabion, Pfizer, Roche, and UCB SA, Timothy Radstake Shareholder of: AbbVie, Employee of: AbbVie, Su Chen Shareholder of: AbbVie, Employee of: AbbVie, Dilek Arikan Shareholder of: AbbVie, Employee of: AbbVie, Hartmut Kupper Shareholder of: AbbVie, Employee of: AbbVie, Howard Amital Consultant of: Abbvie, Janssen, Novartis, Roche, Perrigo Pfizer, Neopharm, Elly Lilly, Gilead, Sanofi, Teva and Rafa, Grant/research support from: Yansen, Pfizer DOI: 10.1136/annrheumdis-2021-eular.221

\section{OP0116 RISK OF SERIOUS INFECTIONS WHEN RECEIVING BDMARDS OR JAK INHIBITORS COMPARED TO CSDMARD TREATMENT}

A. Strangfeld ${ }^{1}$, B. Manger ${ }^{2}$, M. Worsch ${ }^{3}$, T. Schmeiser ${ }^{4}$, A. Zink ${ }^{1}$, M. Schaefer ${ }^{1}$ ${ }^{1}$ German Rheumatism Research Centre, Epidemiology and Health Care Research, Berlin, Germany; ${ }^{2}$ University Hospital Erlangen, Faculty of Medicine, Erlangen, Germany; ${ }^{3}$ Private Practice, Mühlhausen, Germany; ${ }^{4}$ Private Practice, Cologne, Germany

Background: Elderly rheumatoid arthritis (RA) patients are generally at increased risk of serious infections (SI). At the same time, treatment with bDMARDs has been associated with a higher SI risk than treatment with csDMARDs (1). However, long-term use of bDMARDs did not increase the risk of $\mathrm{SI}$ in a small group of elderly patients over 65 (2). The extent to which elderly patients are exposed to a higher SI risk when treated with JAK inhibitors (JAKi) is an open question. Objectives: To assess the effects of bDMARDs and specifically JAKi on the risk of $\mathrm{SI}$ in elderly patients with RA.

Methods: The German register RABBIT is a prospective, longitudinally followed cohort of RA patients enrolled with a new start of a DMARD after at least one csDMARD failure. This analysis comprises patients over 70 years of age who were enrolled between 01/2007 and 04/2020 and had at least one follow-up. 